\title{
Systemic thromboembolic adverse events in patients treated with intravitreal anti-VEGF drugs for neovascular age-related macular degeneration: an update
}

Ciro Costagliola, Francesco Morescalchi, Sarah Duse, Davide Romano, Giuseppina Mazza, Francesco Parmeggiani, Silvia Bartollino \& Francesco Semeraro

To cite this article: Ciro Costagliola, Francesco Morescalchi, Sarah Duse, Davide Romano, Giuseppina Mazza, Francesco Parmeggiani, Silvia Bartollino \& Francesco Semeraro (2019): Systemic thromboembolic adverse events in patients treated with intravitreal anti-VEGF drugs for neovascular age-related macular degeneration: an update, Expert Opinion on Drug Safety, DOI: $10.1080 / 14740338.2019 .1643838$

To link to this article: https://doi.org/10.1080/14740338.2019.1643838

Accepted author version posted online: 16 Jul 2019. Published online: 19 Jul 2019.

Submit your article to this journal $₫$

凹ll Article views: 18

View Crossmark data \lceil 


\title{
Systemic thromboembolic adverse events in patients treated with intravitreal anti-VEGF drugs for neovascular age-related macular degeneration: an update
}

\author{
Ciro Costagliola ${ }^{a}$, Francesco Morescalchi ${ }^{\text {}}$, Sarah Duse ${ }^{\mathrm{b}}$, Davide Romano ${ }^{\mathrm{b}}$, Giuseppina Mazza ${ }^{\mathrm{b}}$, \\ Francesco Parmeggianic ${ }^{c}$, Silvia Bartollino ${ }^{a}$ and Francesco Semeraro ${ }^{b}$
}

\begin{abstract}
aDepartment of Medicine and Health Science "V. Tiberio", University of Molise, Campobasso, Italy; bepartment of Medical and Surgical Specialties, Radiological Sciences and Public Health, University of Brescia, Brescia, Italy; 'Department of Morphology, Surgery and Experimental Medicine, University of Ferrara, Ferrara, Italy
\end{abstract}

\section{ABSTRACT}

Introduction: Intravitreal anti-VEGF is the most effective therapy for wet AMD, although systemic effects on the endothelium cannot be excluded.

Areas covered: The purpose of this review was to evaluate risk of thromboembolic events associated with intravitreal anti-VEGF.

Expert opinion: Current data are insufficient to confirm the safety of these compounds, due to the paucity of specific studies. Thus, pharmacovigilance for all anti-VEGF should be improved to verify the true role of anti-VEGF in the occurrence of systemic adverse events.
ARTICLE HISTORY

Received 6 June 2019

Accepted 11 July 2019

\section{KEYWORDS}

Abicipar; aflibercept; age-related macular degeneration; anti-VEGF; bevacizumab; conbercept; ranibizumab; thromboembolic adverse events

\section{Introduction}

Age-related macular degeneration (AMD) is the most common cause of progressive and irreversible vision loss in adults aged 55 years and older in developed countries. It varies by ethnicity, occurring more frequently in the Caucasian race and very rarely in the African race. Among modifiable AMD risk factors, there are smoking, lack of physical exercise, a diet poor in carotenoids, omega-3 fatty acids, antioxidants, and vitamins [1].

The estimated current worldwide prevalence of AMD is about 170 million and by 2040, it could rise to 288 million [2].

Early-AMD is characterized by hipo and/or hyperpigmentation of the retinal pigmentary epithelium (RPE) and drusen; late AMD is characterized by photoreceptors, RPE, and choroidocapillaris' atrophy (dry-AMD) or choroidal neovascularization (CNV) within the macula (wet-AMD).

CNV in wet-AMD accounts for most cases of AMD-related severe vision loss.

There are no effective therapies for dry-AMD; however, since 2006, intravitreal anti-vascular endothelial growth factor (anti-VEGF) drugs have completely revolutionized the management of wet-AMD, and their efficacy and safety have been validated through a great number of clinical studies [3].

The intravitreal anti-VEGF therapy for wet-AMD has improved the quality of life of millions of patients around the world, and millions of patients have used the intraocular VEGF blockade therapy for many months or years.

The blood retinal barrier is effective in preventing the access of serum plasma contents into the eye, but it does not prevent a part of the injected drugs from seeping into the bloodstream. Thus, the repetition of anti-VEGF therapy can lead to a significant decrease of VEGF serum concentration. Circulating VEGF is important for protecting the integrity and the patency of vessels in many tissues, including the myocardium and the brain.

The inhibition of circulating VEGF is associated with the reduction of the production of nitric oxide (NO) and prostaglandin-I (PG-12) 2, as well as the degeneration and death of the vasal endothelial cells. The reduction in the concentrations of NO and PG-12 causes a peripheral vasoconstriction with consequent hypertension. The degeneration of endothelial cells causes the exposure of phospholipids and extracellular matrix to pro-coagulant action in the lumen of the vessels, favoring thrombosis (cerebrovascular accidents, myocardial infarction, transient ischemic attacks, etc.) [4].

Prolonged VEGF inhibition may increase the risk of stroke, myocardial infarction, and thromboembolic events, thereby raising the issue of safety. In this review, we discuss the safety data that have emerged from all the trials involving the four main anti-VEGF drugs: pegaptanib, ranibizumab, bevacizumab, and aflibercept and report the data available on the recently introduced anti-VEGF drugs: conbercept and abicipar pegol.

\section{Anti-VEGF compounds and their pharmacokinetics}

\subsection{Pegaptanib}

Pegaptanib sodium (Macugen ${ }^{\circledR}$; EyeTech, New York, NY) is a pegylated aptamer, a small RNA fragment with a molecular weight of $50 \mathrm{kDa}$. It is the only anti-VEGF drug that is designed 


\section{Article highlights}

- Anti-VEGF agents represent a turning point in the treatment of AMD in terms of efficacy.

- Arterial thrombotic risk appears sufficiently low when compared with the natural incidence of arterial-thrombotic events in elderly patients and is acceptably balanced against the advantage of improved vision.

- Rates of serious cardiovascular adverse events are similar in patients treated with different anti-VEGF drugs.

- All patients undergoing treatment with anti-VEGF agents, however, require long-term general monitoring.

This box summarizes key points contained in the article.

to bind only the $\mathrm{VEGF}_{165}$ isoform at its heparin-binding domain, blocking its interaction with its receptors on endothelial cells.

The FDA approved Pegaptanib for the treatment of neovascular AMD in 2004 [5].

Pegaptanib remains in the vitreous for a long time before it passes into systemic circulation, where it is quickly cleared through renal metabolism and excretion.

In the eye, it penetrates all retinal layers and can be detected for up to 10 days in the plasma [6]. In humans, one intravitreal injection of $3 \mathrm{mg}$ Pegaptanib gives a mean plasma level of $80 \mathrm{ng} / \mathrm{ml}$, with a plasma half-life of 10 days [7]. After the initial positive experiences with Pegaptanib, it proved to be less effective than the other 'pan-VEGF' inhibitors, and now it is rarely chosen for treating wet-AMD.

\subsection{Ranibizumab}

Ranibizumab (Lucentis ${ }^{\oplus}$; Novartis Pharma AG, Basel, Switzerland, and Genentech Inc., South San Francisco, CA, USA) is a recombinant humanized immunoglobulin G1 kappa isotype antibody fragment with a molecular weight of $48 \mathrm{kDa}$. It can bind all VEGF-A isoforms and block the signaling pathway. It does not contain the Fc antibody region, and therefore it is cleared from bloodstream faster than other anti-VEGF drugs and has a short systemic elimination half-life of $2 \mathrm{~h}$. In June 2006, ranibizumab was approved for the treatment of AMD [8,9].

Its vitreal half-life is approximately 9 days. Pharmacokinetics (PK) studies have demonstrated that ranibizumab does not accumulate systemically between two injections. Its serum half-life after intravitreal administration is $5.8 \pm 1.8$ days. After two repeated injections, ranibizumab serum levels exceeded its reported $I_{50}(0.060 \mathrm{nM})$ for almost $24 \mathrm{~h}$. In patients who received ranibizumab for AMD treatment, no notable changes were observed in the mean free-VEGF levels from baseline after injection [10].

\subsection{Bevacizumab}

Bevacizumab (Avastin ${ }^{\circledR}$, Genentech, Inc, South San Francisco, CA), is a humanized, function-blocking, monoclonal murine antibody that has a molecular weight of $149 \mathrm{kDa}$. Bevacizumab was the first VEGF inhibitor approved by the FDA for systemic use in colorectal cancer (CRC), advanced non-small cell lung carcinoma
(NSCLC), metastatic breast cancer (MBC), advanced renal cell cancer and as second-line treatment for glioblastoma multiforme and other solid tumors. Bevacizumab is also effective in treating wet-AMD when injected intravitreally at a dose of $1.25 \mathrm{mg}$; however, it can only be used off-label at present. It acts by selectively inhibiting the binding of VEGF to its cell surface receptor. Bevacizumab, when administrated systemically at doses 500 times higher than the intravitreal dose, may cause hypertension, thromboembolic events, gastrointestinal perforation, and wound healing complications. A meta-analysis showed that $11.9 \%$ (95\% Cl 6.8\%-19.9\%) of patients with different malignancies developed thromboembolism (RR 1.33; $95 \% \mathrm{Cl}$ 1.13-1.56; $p<0.001$ ) when treated with high systemic doses of bevacizumab [11].

Bevacizumab is approximately three times larger than ranibizumab (149 kDa vs. $48 \mathrm{kDa}$ ) and, due to it is substantially higher molecular weight, its intravitreal half-life is $75 \%$ more longer; however, it is less than that of Aflibercept [12].

Bevacizumab has the Fc component of the antibody intact; therefore, it penetrates into human retina within $24 \mathrm{~h}$ (slower than ranibizumab) and is actively moved from the eye to the systemic circulation where it can be detected for several weeks.

Heiduschka et al. found that only $5-10 \%$ of the intraocularly injected bevacizumab may be detected in the blood of monkeys, suggesting a peak serum concentration perhaps between 20 and $50 \mathrm{ng} / \mathrm{mL}$ in humans [13]. These findings were confirmed by further studies in humans [14]. After a single intravitreal injection of $1.25 \mathrm{mg}$ of bevacizumab in patients with $A M D$, the maximum serum concentration was found to be between 59.8 and $86.5 \mathrm{ng} / \mathrm{mL}[14,15]$, corresponding to a maximal molar concentration of $0.76 \mathrm{nM}( \pm 0.3) 7$ days after the first dose [10], which is close to its $I_{50}(=0.67 \mathrm{nM})$, the concentration necessary to inhibit $50 \%$ of the biological activity of serum VEGF [16].

Bevacizumab has a longer systemic half-life in plasma (18-20 days) than ranibizumab (2-6 h) and Aflibercept (1-5 days) and tends to accumulate in the serum, if administered monthly [10].

\subsection{Aflibercept}

Aflibercept (Eylea ${ }^{\circledR}$; Regeneron USA, Tarrytown, NY and Bayer, Switzerland) or VEGF-Trap eye is the latest approved compound in Europe and America, which was derived from the native VEGF receptor. Structurally, Aflibercept is a soluble decoy receptor of $115 \mathrm{kDa}$, which is made by the second binding domain from VEGF-R1 and the third binding domain from VEGF-R2, which are fused to the FC region of a human IgG1. It acts as a competitive inhibitor of VEGF, also binding to placental growth factor 1 and $2[17,18]$.

Aflibercept forms a stable, 1:1 complex with either VEGF-A, VEGF-B, or placental grow factor (PIGF) ligand, inhibiting the activity of both VEGF receptors. Its molar binding activity for VEGF is 140, thus the affinity of Aflibercept for VEGF is 100times higher than that of Bevacizumab and Ranibizumab [17].

The intermediate size of Aflibercept (115 kD), compared to $48 \mathrm{kD}$ of Ranibizumab and $148 \mathrm{kD}$ of Bevacizumab, leads to prolonged clinical action inside the eye, possibly as long as 2.5 
months, exceeding the 1-month intravitreal binding activity of Ranibizumab. After the intravitreal injection of Aflibercept (2 mg), it was detected in plasma, mostly as an inert complex bound to VEGF, and thus inactive, and in minor quantities as an active, free drug.

The mean maximum concentration of free Aflibercept in the plasma was $0.02 \mu \mathrm{g} / \mathrm{ml}$ (range, $0-0.054 \mu \mathrm{g} / \mathrm{ml}$ ) and this concentration was achieved in 1 to 3 days $[14,15]$.

After a single intravitreal injection of Aflibercept $(2 \mathrm{mg}$ ) in AMD patients, the maximum serum molar concentration of Aflibercept was between $0.05( \pm 0.02)$ and $0.47( \pm 0.29) \mathrm{nM}$. The maximum serum concentration often exceeds the IC50 of Aflibercept, which is $0.068 \mathrm{nM}$ [10]. However, most of the drug is inert because it forms a complex with the antigen.

The systemic half-life of unbound Aflibercept is 1.5 days, much lower than that of Bevacizumab (20 days) and closer to that of Ranibizumab ( $6 \mathrm{~h}$ ). The free Aflibercept plasma concentrations were undetectable 2 weeks post-dosing in all patients. Aflibercept did not accumulate in the plasma during the loading phase, but was rapidly cleared from circulation, through a same pathway similar to that for antibody clearance $[18,19]$.

\subsection{Conbercept}

Conbercept (Lumitin ${ }^{\oplus}$; Chengdu Kanghong Biotech Co, Ltd, Chengdu, China) is a new anti-VEGF agent, currently approved only in China. It is a $141 \mathrm{kDa}$ recombinant fusion protein of the VEGF receptor domains with the Fc fragment of human immunoglobulin. The difference between Conbercept (141 kDa) and Aflibercept (115 kDa) is the presence of the fourth binding domain of VEGFR2 in Conbercept, which stabilizes the receptor-ligand complex, extending its half-life and potentially, thanks to its larger molecular size, reducing the blood-ocular barrier permeability. Its presence in the plasma can be detected $4 \mathrm{~h}$ after ITV administration and concentration reaches the maximum (Cmax) of about $5 \mathrm{ng} / \mathrm{mL} 34 \mathrm{~h}$ after dosage (Tmax); it is hardly detected in serum 15 days after dosage. The half-life is approximately 4-5 days [20-22].

\subsection{Abicipar pegol}

Another new anti-VEGF agent is Abicipar Pegol (AGN-150,998, MP0112, Abicipar ${ }^{\oplus ;}$ Allergan plc/Molecular Partners), a member of DARPin ${ }^{\circledR}$ therapeutics (a registered trademark of Molecular Partners AG, Switzerland; DARPin was originally derived from designed ankyrin repeat protein). DARPins are a class of protein binding molecules, already used in oncology, allergic asthma, etc [23-25]. Abicipar binds with high affinity to all soluble isoforms of VEGF-A. Comparable to Ranibizumab, which also binds to all the isoform of VEGF-A, it has smaller molecular weight (34 kDa vs. $48 \mathrm{kDa}$ ), higher affinity (2 pM vs. $46 \mathrm{pM}$ ), and a longer ocular half-life (13 days vs. 7 days).

In the REACH study, at 1 week after IVT administration of $2 \mathrm{mg}$ of Abicipar, the serum concentration of Abicipar was 0.43-0.33 nM. At all other time points, serum concentrations of free Abicipar were below the limit of quantitation $(0.3 \mathrm{nM})$ in all samples [26-29].
SEQUOIA and CEDAR are two identical phase III, multicenter, randomized, ongoing studies designed to evaluate the efficacy and safety of Abicipar Pegol compared to that of Ranibizumab in the treatment of naïve patients with wetAMD. The primary endpoint was the percentage of patients with visual stability at week 52: these studies are still ongoing, and the two-year results are expected in the next few months. In both studies, over the first year, Abicipar showed an efficacy rate comparable to 13 Ranibizumab injections, only after six or eight injections. Overall adverse events were similar between the three treatment arms.

\section{Evidence on thromboembolic complications of anti-VEGF drugs used for wet AMD}

\subsection{Pegaptanib}

The use of Pegaptanib as an anti-VEGF drug for wAMD was first tested in 2004 by Groudas et al., who designed the VISION study, a two concurrent, prospective, randomized, doubleblind, multicentre, study [30]. In this study, 1186 patients were enrolled and randomized into four treatment arms: intravitreal injection of Pegaptanib (at doses of $0.3 \mathrm{mg}, 1.0 \mathrm{mg}$, and $3.0 \mathrm{mg}$ ) and sham injection. Injections were administered every 6 weeks over a period of 48 weeks. The authors did not report systemic adverse events in the different arms of treatments at the end of 48 weeks.

A subsequent follow-up study at 2 years, which was conducted by Chakravarty et al., included 425 patients who were previously enrolled in the VISION study. Those who were initially assigned to pegaptanib were re-randomized $(1: 1)$ to continue or discontinue therapy for 48 more weeks (eight injections). Those initially assigned to the sham group were re-randomized to continue sham, discontinue sham, or receive 1 of 3 pegaptanib doses. The results showed no differences at year 2 in cardiovascular accidents [31].

Of the 161 subjects receiving active therapy (every 6 weeks) with pegaptanib for 3 years, none experienced arterial thromboembolic events (ATE). Only two subjects experienced myocardial infarction (2\%) and one patient had angina (1\%). The incidence of these thromboembolic events was the same among a cohort of 422 subjects treated with pegaptanib for 3 years. The types and incidence of systemic serious adverse events observed are not unexpected in the elderly patient population, and none of these events was judged to be related to the study drug [32].

The safety of the selective VEGF 165 blocker, Pegaptanib, could be due to its inability to interfere with some isoforms of VEGF like VEGF 21 and VEGF 189 which exert physiological action [33,34]. However, in VISION, study patients with a history of myocardial infarction or stroke or even patients with peripheral vascular diseases were excluded [35,36].

A retrospective analysis published in 2018 evaluated the safety of Pegaptanib in patients with a known history of cardiovascular events. Twenty-three patients with subfoveal CNV due to AMD treated with pegaptanib $0.3 \mathrm{mg}$ in the previous 6 months were followed up for 12 months. None of the recruited patients experienced any ATE during the 6 plus 12-month period. This result, according to some authors, 
could suggest the safety of this anti-VEGF in patients at high risk of recurrent ATEs. Some concerns were raised regarding the concomitant anticoagulant and cardiac medication contributing to the lack of further ATE in these patients during the treatment period with pegaptanib [37].

\subsection{Ranibizumab}

Ranibizumab is the most extensively studied anti-VEGF drug that is in widespread use and has been approved by the FDA for treatment of exudative AMD [38].

The firsts studies on Ranibizumab were the minimally classic/occult trial of the anti-VEGF antibody ranibizumab in the treatment of the neovascular AMD (MARINA) [8] and ANCHOR [9].

The MARINA study compared 24 monthly intravitreal injections of ranibizumab $0.3 \mathrm{mg}$ or $0.5 \mathrm{mg}$ versus sham injections in patients with CNV. A total of 716 patients were enrolled in this study, and at 2-year follow up, the percentage of ATEs was slightly higher $(4.6 \%$ vs. $3.8 \%)$ than in the sham injection group, and the incidences of myocardial infarction and stroke were $1.3 \%$ vs. $2.5 \%$, respectively.

In the ANCHOR trial, 423 patients with CNV were randomized at a ratio of 1:1:1 to receive monthly intravitreal injections of ranibizumab $(0.3 \mathrm{mg}$ or $0.5 \mathrm{mg})$ plus sham verteporfin therapy or monthly sham injections plus active verteporfin therapy.

The incidence of arterial thromboembolic adverse events was $5 \%$ in the ranibizumab $0.5 \mathrm{mg}$ group; $4.4 \%$ in the ranibizumab $0.3 \mathrm{mg}$ group; and $4.2 \%$ in the active verteporfin group. Myocardial infarction and stroke occurred in the $3.6 \%$ (ranibizumab) vs. 0\% (verteporfin).

After MARINA and ANCHOR, other studies tried to demonstrate that flexible, guided dosing with fewer ranibizumab injections and monthly monitoring may maintain efficacy outcomes [39].

In the FOCUS trial, 162 patients were randomized into two arms of treatment: intravitreal $0.5 \mathrm{mg}$ ranibizumab every 4 weeks or sham injections every 4 weeks. Both of the treatment arms received PDT at day 0 and then every 12 weeks as required. The incidences of ATEs were slightly higher in the PDT group than in the ranibizumab group during the 2-year period: $7.1 \%$ vs. $4.8 \%$ [40].

In the PIER study, 184 patients were enrolled and randomized at a ratio of 1:1:1 into $0.3 \mathrm{mg}$ and $0.5 \mathrm{mg}$ ranibizumab or sham treatment groups. Ranibizumab had been given quarterly (every 3 months) for 2 years (an average of six injections). At year 2, the incidence of ATEs was $1.7 \%$ in the $0.3 \mathrm{mg}$ group, $0 \%$ in the $0.5 \mathrm{mg}$ group, and $1.7 \%$ in the sham group [41].

In the EXCITE study, 353 patients were randomized at the ratio of 1:1:1 into $0.3 \mathrm{mg}$ quarterly, $0.5 \mathrm{mg}$ quarterly, or $0.3 \mathrm{mg}$ monthly doses of ranibizumab for 1 year. The incidence of ATEs was $2 \%$ in the study with that among the treatment arms differing as follows: $3.5 \%$ in the $0.3 \mathrm{mg}$ monthly dosing regimen; $1.7 \%$ in the $0.3 \mathrm{mg}$ quarterly dosing regimen; and $0.8 \%$ in the $0.5 \mathrm{mg}$ quarterly dosing regimen treatment groups [42].

In the SUSTAIN study, 513 patients, who never experienced intravitreal ranibizumab, were administered three initial monthly injections of ranibizumab $(0.3 \mathrm{mg})$ and, thereafter, as needed (pro re nata, PRN), retreatment for 9 months based on pre-specified retreatment criteria. Patients switched to $0.5 \mathrm{mg}$ ranibizumab after its approval in Europe [39].

ATEs were reported in $3.7 \%$ of patients, with $1.0 \%$ experiencing cerebrovascular adverse events, whereas the total rate of systemic adverse events was $14.2 \%$.

About $10 \%$ of patients who had a stroke earlier suffered another stroke in the first 12 months after initiation of therapy, showing that ranibizumab could potentially cause a slight increase in stroke risk $[43,44]$. The rate of adverse systemic events that emerged in the SUSTAIN study was almost similar to that observed in patients treated with $0.5 \mathrm{mg}$ intravitreal ranibizumab in the MARINA and ANCHOR studies, in which the rates were $4.4 \%$ and $5 \%$ of patients, respectively $[8,9,45]$. However, a direct comparison of the safety data from SUSTAIN and the other previously published studies is not entirely possible for several reasons, such as different treatment regimens, different classification systems for adverse events, different study design, and the fact that the patients enrolled in the SUSTAIN were not naive.

The SAILOR study was a large phase Illb follow-up study to the MARINA and ANCHOR studies to evaluate the long-term safety and efficacy of ranibizumab in a population of 4300 patients with all subtypes of CNV (predominantly classic, minimally classic, and occult without classic). SAILOR included more than five times as many ranibizumab-treated patients as the MARINA and ANCHOR studies combined [46]. Thus, it is the largest multicentre-randomized study, to date, that evaluated the safety and efficacy of anti-VEGF treatment in wet AMD. After 1 year, arterial-thrombotic events were reported in $2.8 \%$ of patients, following an average of 4.6 injections. The SAILOR study had two cohorts. Cohort 1 subjects were randomized to receive $0.3 \mathrm{mg}(\mathrm{n}=1169)$ or $0.5 \mathrm{mg}(\mathrm{n}=1209)$ intravitreal ranibizumab for 3 monthly loading doses. Cohort 2 subjects $(n=1922)$ received an initial intravitreal dose of $0.5 \mathrm{mg}$ ranibizumab and were retreated at the physician's discretion. Safety was evaluated at all visits. The incidence of vascular deaths during the 12 -month study was $0.9 \%$ in the cohort $10.3 \mathrm{mg}$ group, $0.8 \%$ in the cohort $10.5 \mathrm{mg}$ group, and $0.7 \%$ in cohort 2 , respectively. The rates of individual key ocular serious adverse events in both cohorts were less than $1 \%$. The number of vascular deaths, as well as deaths due to unknown causes, did not differ across the cohorts or dose groups. Stroke rates were $0.7 \%, 1.2 \%$, and $0.6 \%$ in the $0.3 \mathrm{mg}$ and $0.5 \mathrm{mg}$ groups and cohort 2, respectively.

However, in February 2007, the FDA observed that the rate of stroke in both dose arms of the SAILOR study was lower than that in the approval studies, suggesting that it may have underestimated the systemic effects or selected patients in good health and without significant cardiovascular risk [8].

An interim analysis of SAILOR cohort 1 safety data (October 2006) suggested a higher incidence of stroke in subjects who received $0.5 \mathrm{mg}$ ranibizumab compared with those who received $0.3 \mathrm{mg}$ ranibizumab. The final study data showed a difference in stroke rate between doses, with a higher rate in the $0.5 \mathrm{mg}$ dose group than in the $0.3 \mathrm{mg}$ dose group. The total number of events was small, and the difference was not confirmed statistically. However, 
there is potentially a higher risk of stroke associated with the $0.5 \mathrm{mg}$ dose, which is being monitored via post-marketing surveillance and ongoing trials of ranibizumab in neovascular AMD. Certain subgroups of subjects (e.g. those with prior cardiovascular accidents) may experience higher rates of systemic adverse events. A meta-analysis of ranibizumab systemic safety, based on a pooling of first-year data from the MARINA, ANCHOR, PIER, FOCUS, and SAILOR trials and on second-year data from the MARINA, ANCHOR, PIER, and FOCUS trials showed that arterial thromboembolic events rates were similar across the treatment groups, although the rates of side effects appeared to be slightly higher in those treated with higher doses of ranibizumab than in the controls.

These analyses suggested that in persons with prior stroke and in those with arrhythmia, stroke risk was higher in those treated with higher doses of ranibizumab $[47,48]$.

Further analysis on these trials, on the 859 subjects who were injected monthly for 2 years, showed that ranibizumab was associated with higher incidence of stroke ( $p=0.045$; OR, $3.24 ; 95 \% \mathrm{Cl}, 0.96-10.95)$, whereas there was no apparent association with the incidence of myocardial infarction ( $p=$ 0.193) [49]. Thus, the central nervous system seems more sensitive than the cardiovascular system to even a small amount of ranibizumab injected into the vitreous body.

HORIZON was an open-label extension trial of ranibizumab for the treatment of neovascular AMD. Only those patients who were previously enrolled in the MARINA, ANCHOR, and FOCUS trials and who had completed a 2-year follow up were re-randomized to receive $0.5 \mathrm{mg}$ ranibizumab or sham injections for 24 months. The treatment was administered at the investigator's discretion (a mean of 4.4 injections were administered for each patient). The aim of the study was to explore the effects of ranibizumab after 2 years of treatment. A total of 84 patients had completed 2 years of follow-up; therefore, data were available on 4-5 years of ranibizumab treatment. There was a decline in vision (with a mean of minus seven letters along with the follow-up) after the reduction of the monthly frequency of the injections [50]. The incidence of ATEs was similar among the two treatment groups: $5.3 \%$ in 0.5 ranibizumab arm versus $3.2 \%$ in the sham arm.

In the SECURE study, ranibizumab was administered according to the treatment strategy adopted in Europe (visual acuity-guided flexible dosing regimen) over a follow-up period of 2 years [51].

The study was a phase IV extension study in which 210 patients previously included in the EXCITE and SUSTAIN studies were treated for up to 3 years with ranibizumab $[39,42]$.

Patients from the EXCITE and SUSTAIN trials were treated for 1 year with variable dosing regimens (quarterly or PRN) of Ranibizumab.

In the SECURE study, after a mean of 6.1 injections over 2 years (apart from those received injections in the EXCITE and SUSTAIN studies), ATEs, including hemorrhagic cerebrovascular conditions, myocardial infarction, and ischemic cerebrovascular disease, were recorded in $5.6 \%$ of the patients. Furthermore, similar to what was observed in the HORIZON study, the reduction in the frequency of intravitreal injection caused a decline in vision. A statistically significant increase in systolic and diastolic blood pressure was recorded in $11.5 \%$ and $3.6 \%$ of the patients, respectively.

The HELIOS STUDY was a prospective, multicenter study, in which $0.5 \mathrm{mg}$ ranibizumab was administered to 267 patients affected by wet AMD, and the patients were followed up for 2 years. This study, conducted in Belgium, analyzed the use of ranibizumab in clinical practice for the management of neovascular AMD and is a part of the pharmaco-vigilance studies about this compound.

The safety of ranibizumab in terms of the percentage of systemic adverse events was comparable to the product label listings. The authors reported a total of 78 systemic adverse events, including 24 cardiovascular events (9.4\%), six systemic events, and one gastrointestinal event, in 40 out of 255 patients. Only nine of these systemic adverse events were related to the ranibizumab treatment [52]. One of the limitations of this study was that the TAEswere not separately classified. However, this study classified the patients as non-smokers, smokers or former smokers. Cigarette smoke, which is recognized as a major risk factor for cardio-and cerebrovascular disease, could, therefore, represent a confounding factor in the analysis of thromboembolic risk related to this drug. In the study population, the percentage of current and former smokers was about $43.6 \%$, which is very similar to that of the Belgian population (51\%) [53].

Other contributions to the definition of the systemic safety of ranibizumab will be given by the LUMINOUS study, which is a multicenter study recently initiated as a part of a pharmacovigilance program by Novartis to assess the longterm safety and quality-of-life of patients treated with intravitreal Lucentis. The study plans to enroll about 30,000 patients from Europe, Asia, Australia, and America and follow up with them for 5 years [54]. The LUMINOUS study has retrospective and a prospective parts, thus it includes, in addition to the above-mentioned observational study, a retrospective safety study on ranibizumab. The retrospective analysis will be conducted on the basis of observations from 1-year data from four European ranibizumab registries (from Germany, Netherlands, Belgium and Sweden). Data collected from 4444 patients will provide an insight into the systemic safety of the drug.

In the LUMINOUS study, the risk of cerebrovascular incidence and the annual incidence of stroke was found to be $0.43 \%$; this percentage is lower than that obtained with $0.5 \mathrm{mg}$ ranibizumab in the SAILOR study (1.2\%). However, the mean number of injections per patient in the analysis was very low (4.3) in comparison to that in the SAILOR study (4.6 in cohort 1) and in the SUSTAIN study (5.6), and it was also lower than that in the ranibizumab as-needed arm of the CATT study at 1 year (6.9) [46].

Even in the WAVE (Lucentis in Wet AMD: Evaluation of Visual Acuity and Quality of Life) study, a multicentric non interventional study conducted in Germany, which investigated the quality of life of 3470 patients affected by wetAMD, the rate of recorded strokes was low $(0.4 \%)$, with a mean of 4.3 intravitreal injection that could have underestimated its incidence [55]. 
Indeed, a common experience of all ophthalmologists is that four injections are rarely sufficient to stop the progression of wet AMD. Most of the visual acuity improvement acquired after the loading phase of 3 months can be eroded after transition to less frequent dosing.

Vision performances and anatomical aspect of the macula were consistently better in the subgroups under monthly regimen than in those under PRN or quarterly regimen. A metaanalysis promoted by Ueta et al. aimed to compare the safety levels among the various Ranibizumab regimen categories (0.5 mg vs. sham; $0.5 \mathrm{mg}$ vs. $0.3 \mathrm{mg}$; monthly versus PRN; $0.3 \mathrm{mg}$ versus sham) and among combined regimen categories $(0.3 \mathrm{mg} / 0.5 \mathrm{mg}$ versus sham, $0.5 \mathrm{mg}$ vs. $0.3 \mathrm{mg} / \mathrm{sham}$, and monthly versus PRN/control treatments). Authors concluded that there is neither a significant increase in the risk of myocardial infarction nor ATEs among all the categories of ranibizumab regimens. Authors reported an increased risk of the cerebrovascular accidents (CVAs), just in the combined regimen categories of $0.5 \mathrm{mg}$ versus combination of $0.3 \mathrm{mg}$ and no ranibizumab groups $(\mathrm{OR}, 1.86 ; 95 \% \mathrm{Cl}, 1.05-3.29 ; p=$ 0.03 ) and monthly treatment group versus the combined PRN and no ranibizumab groups (OR, $1.89 ; 95 \% \mathrm{Cl}, 1.06-3.38 ; p=$ 0.03). According to the authors, the increased risk of CVAs, may be due to anatomic proximity of the vitreous cavity and the subarachnoid space [56].

\subsection{Bevacizumab}

The FDA originally approved bevacizumab in 2004 for chemotherapy, in particular for the treatment of metastatic colorectal cancer but not for the treatment of eye conditions. Originally, it was manufactured for intravenous use in oncology; however, it was soon also used in ophthalmology. Intravitreal injections of bevacizumab are produced by splitting doses of the cancer drug and making numerous unit doses from a vial of bevacizumab.

The 'off-label' or unlicensed use of bevacizumab in the treatment of wet-AMD emerged in the United States in 2005, and rapidly became a widespread practice among ophthalmologists worldwide. The use of bevacizumab is attractive in comparison with other anti-VEGF agents because of its low cost considering the number of injections, which are necessary at 4- to 6-week intervals.

Despite its off-label status, bevacizumab is widely used by most physicians worldwide because of the evidence of its efficacy that emerged from many clinical reports and several randomized controlled clinical trials. Additionally, its affordability makes it the drug of choice for thousands of patients who would have otherwise not had access to anti-VEGF drugs because of their high cost [57].

Systemic adverse effects have been a long-standing area of debate for intravitreal off-label use of bevacizumab [58]. The first large-scale study with one-year follow-up was conducted by $\mathrm{Wu}$ and colleagues in 2008. They treated 1173 patients with bevacizumab intravitreally and reported a low systemic side effect rate in 18 patients (1.5\%). Among them, there were seven hypertensive crisis $(0.59 \%)$, six cerebrovascular accident $(0.5 \%)$, five myocardial infarction $(0.4 \%)$, and five deaths $(0.4 \%)$ cases [59].
The larger retrospective non-randomized study was performed in 702 patients treated in Seoul National University Hospital. Bevacizumab was administered to 610 patients and ranibizumab to 240 patients for 1 year. This study found that systemic adverse events occurred in seven of the 503 patients (1.4\%) in the bevacizumab group and in three of the 199 patients in the ranibizumab group (1.5\%) without a significant difference [60].

The first prospective comparative case series was carried out on 120 patients in the Eye Institute in Kolkata (India). The comparison of the efficacy and safety of intravitreal ranibizumab and bevacizumab showed similar improvement in vision, no thromboembolic events, and similar safety profiles [61].

An important contribution was provided by a large comparative retrospective study conducted on 146,942 Medicare and Medical Services beneficiaries (with wet-AMD), who received intravitreal pegaptanib, bevacizumab, ranibizumab, or photodynamic therapy. After adjusting for patient characteristics and economic status and limiting the comparison to newly treated patients in order to alleviate potential selection bias, significant differences were not observed in safety outcomes between the bevacizumab and ranibizumab groups.

The cumulative incidence at 1 year of myocardial infarction and stroke, respectively, was $1.3 \%$ and $2 \%$ in the photodynamic therapy group, $1.3 \%$ and $2 \%$ in the group treated with pegaptanib, $1.2 \%$ and $2.1 \%$ in the group treated with bevacizumab, and $1.1 \%$ and $1.8 \%$ in the group treated with ranibizumab.

All patients were older than 65 years of age and no evident difference was found in stroke hazard, myocardial infarction, or mortality between bevacizumab use and other on-label therapies [62-65].

Another vast population nested case-control study has been conducted in Canada, including 91,378 older adults with retinal disease. The authors identified 7,388 serious cardio-circulatory events, including ischemic stroke (1.6\%), myocardial infarction (2.4\%), venous thromboembolism (1.2\%), and congestive heart failure (2.9\%). Controls were chosen on the basis of the common year of birth, sex, history of the outcome, and diabetic status. The authors found that patients affected by these cardio-circulatory events were not more likely than controls to have been exposed to either Bevacizumab or Ranibizumab. Further analysis revealed that patients treated with bevacizumab or ranibizumab showed no clinically important increase in the risk for ischaemic stroke, myocardial infarction, venous thromboembolism, and congestive heart failure [66].

However, recently the published data from three randomized controlled trials that have directly compared ranibizumab and bevacizumab in patients with wet AMD were made available: the CATT, Inhibit VEGF in Age-related choroidal Neovascularisation (IVAN), and GEFAL studies [67-69].

The CATT is a multicentre randomized, controlled trial conducted in the USA, including 1,208 patients with a median age of 80 years affected by AMD. The patients were randomized to four groups of treatment: Bevacizumab and Ranibizumab, administered every month and bevacizumab and ranibizumab, given 'as needed' or 'PRN.' The number of intravitreal injections for the as-needed regimen groups performed in the 
CATT were 7.7 (bevacizumab) and 6.9 (ranibizumab) injections per year, whereas the number was 13 in monthly dosing groups.

The number of patients affected by ATEs was similar in the Bevacizumab-treated patients (5.0\%) and in the Ranibizumabtreated patients $(4.7 \% p=0.89)$. In addition, the proportion of venous thrombotic events were not significantly different, occurring in $1.7 \%$ of Bevacizumab-treated patients and in $0.5 \%$ of Ranibizumab-treated patients ( $p=0.054$ ).

The rate of general serious adverse events for Bevacizumab-treated patients was higher either in year 1 or year 2, with increased the relative risk of 1.30; however, these events mostly appeared to be not specifically due to the anti-VEGF therapy and perhaps due to chance or imbalances at baseline not captured in multivariate modeling [67].

A new finding of this trial comprised the appearance or the enlargement of the area of geographic atrophy behind and around the CNV after the anti-VEGF treatment.

The development of geographic atrophy was mostly evident in monthly-treated patients than in the as needed treated patients ( $34 \%$ vs. $26 \%, p=0.03$ ). The Ranibizumab monthly treated group displayed the highest probability to develop geographic atrophy than any of the other treatment groups, thus serving as an important clinical consideration to be taken in account when the number of injection increases and explains the common trend to a progressive decline of visual acuity in all the trials.

Of the patients enrolled in CATT, 71\% completed the 5-year follow-up and the ATEs incidence was reported to be suddenly and significantly changed. It was $7.6 \%$ in the Ranibizumab and $4.5 \%$ in patients originally assigned to Bevacizumab $(p=0.04)$; however, as detailed above, during the 2-year clinical trial, the proportion of patients with these events was nearly equal to $4.7 \%$ of Ranibizumab-treated patients and $5.0 \%$ of Bevacizumab-treated patients having an event.

According to the authors, the 5-year ATE difference could be meaningless due to the lack of Ranibizumab or Bevacizumab exposure after the 2-year treatment period. Thus, the higher ATE in the Ranibizumab arm could be attributed to chance [70].

The IVAN was a multicentre randomized, controlled trial conducted at 23 UK centers including 610 patients affected by AMD that were randomized in a similar manner to that in CATT into four treatment groups: bevacizumab $1.25 \mathrm{mg}$ and ranibizumab $0.5 \mathrm{mg}$, administered in either a continuous monthly regimen or an as-needed regimen.

In the group as needed, after a loading dose of three injections every month, if the requirements for a new injection had met, other three injections were performed every month. At 2 years, no difference was observed between the drugs for any of the primary or secondary efficacy outcomes. Data from this trial demonstrated that bevacizumab was non-inferior to Ranibizumab after 2 years of either continuous or discontinuous treatment with respect to visual acuity.

Although the trials brought forth similar findings at 1 year, the systemic adverse events occurring in the second year in the IVAN trial shifted the 2-year odd ratio almost to one unity.

In terms of safety in the IVAN trial, the rate of an arterialthrombotic event or heart failure hospitalization was not significantly different when comparing Ranibizumab with Bevacizumab (6\% vs. $4 \%$; OR $1.69,95 \% \mathrm{Cl} 0.80-3.57$ ) or continuous with as-needed administration (4\% vs. 7\%; OR 0.56, 95\% Cl 0.27-1.19).

The mortality rate was found to be similar between the two drugs after 2 years, and was lower with continuous treatment showing borderline significance (OR 0.47, 95\% Cl 0.22-1.03).

For both drugs, monthly injection resulted in lower CNV growth and slightly better visual acuity than the as-needed treatment. Furthermore, pooling the results of CATT and IVAN resulted in no differences between the bevacizumab or ranibizumab treatment, in terms of deaths or arterial-thrombotic events at 2 years [71] v.

Ranibizumab treatment was associated with a slightly lower likelihood of resulting in any systemic, serious adverse event than bevacizumab (OR $0.76,95 \% \mathrm{Cl} 0.63-0.93$ ), and continuous administration was associated with a lower mortality rate (OR $0.49,95 \% \mathrm{Cl} 0.27-0.86$ ) and a trend toward a higher risk of any systemic serious adverse event $(p=0.063)$. The researchers went on to state that comparisons between the drugs after 2 years were reassuring, with no suggestions of differences in mortality or arterial-thrombotic events, which have previously been suggested to be related to use of anti-VEGF drugs.

The GEFAL study was conducted in France between June 2009 and November 2011 to compare the efficacy of ranibizumab and bevacizumab. The rate of serious systemic adverse events (SAE) in the two groups of treatment was studied as a secondary outcome. Five hundred and one patients were enrolled ( $n=255$, treated with bevacizumab; $\mathrm{n}=246$ treated with ranibizumab). Moreover, the frequency of SAE (at least one) did not differ significantly between the two groups $(12.6 \%$ of SAE in bevacizumab, $n=31 ; 12.1 \%$ in the ranibizumab group, $\mathrm{n}=29 ; p=0.88$ ). Five deaths occurred during the trial, with two $(0.8 \%)$ occurring in the bevacizumab group (reported causes of death: pulmonary fibrosis and one unknown), three (1.3\%) occurring in the ranibizumab group (reported causes of death: metastatic pancreatic cancer, myocardial infarction, and suicide). According to the investigators, none of the deaths were related to drug administration. The proportions of patients with serious ATEs were similar across the groups [69].

The MANTA study, which was similar to IVAN and GEFAL demonstrated no difference between the rates of ATEs between ranibizumab and bevacizumab, which further substantiated the results of CATT trial [72].

The LUCAS study was another large scale, multicentric clinical trial conducted to compare Lucentis with Avastin in terms of their efficacy and safety. Between March 2009 and July 2012, 441 patients were randomized at 10 ophthalmological clinics in Norway. There were fewer ATEs (1.4\%) in the bevacizumab group than in the ranibizumab $(4.5 \%)(p=$ 0.050), and significantly more cardiac events occurred in patients in the ranibizumab group ( $p=0.036)$. However, more patients treated with ranibizumab often had a history of myocardial infarction $(p=0.021)$ [64].

A meta-analysis evaluated the relative risk of $S A E$ in patients treated with either ranibizumab or bevacizumab. This study used data from bibliographic databases and clinical trial registries published till March 2014 and identified five 
large-scale multicentric clinical trials (CATT, IVAN, GEFAL, LUCAS, and BRAMD). Cumulative meta-analysis was conducted on 3052 patients treated with anti-VEGF for nAMD $(n=1513$ treated with bevacizumab, $\mathrm{n}=1539$ treated with ranibizumab). Four hundred and three (26.6\%) patients in the bevacizumab arm and $366(23.8 \%)$ in the ranibizumab who developed SAE. RR of SAE in patients treated with bevacizumab was $1.06,95 \% \mathrm{Cl}(0.84-1.35)$. Arteriothrombotic events, as defined by Antiplatet Trialists' Collaboration (APTC), were lower in patients treated with bevacizumab than in those treated with ranibizumab but with $95 \%$ IC spanning (RR = 0.89, 95\% Cl 0.62-1.28) [73].

An observational study conducted in Thailand by Sangroongruangsri et al. [74] compared the safety profiles of intravitreal bevacizumab and intravitreal ranibizumab, for the treatments of retinal diseases, not just wet-AMD. In total, 6354 patients, between 2013 and 2015, were enrolled in eight hospitals across Thailand; 379 patients were treated with ranibizumab and 5975 patients were treated with bevacizumab, for a total number of 17,395 intravitreal injections, in 6 months (974 injections of ranibizumab and 16,421 injections of bevacizumab). During the 6 months of follow up, 1 case of nonfatal myocardial infarction $(0.26 \%)$ was reported in the ranibizumab arm of treatment, 1 case of heart failure $(0.26 \%), 0$ cases of pulmonary embolism and transient ischemic attack. However, in the bevacizumab group, 27 cases non-fatal myocardial infarction (0.33\%), 17 cases of non-fatal stroke $(0.27 \%)$, 13 deaths from vascular causes $(0.22 \%), 89$ cases of heart failure $(1.21 \%), 1$ case of pulmonary embolism $(0.02 \%)$, and two cases of transient ischemic attack $(0.03 \%)$ were reported.

Considering the sample size in each arm of treatment and the number of adverse events, there was no significant increase in the ATE rate $(95 \% \mathrm{Cl} 0.48-8.01)$ and non-fatal heart failure $(95 \% \mathrm{Cl}$ 0.37-19.60) between the ranibizumab and bevacizumab. Instead, by considering the number of injections, patients who received a higher number of injections ( $>3$ injections) had a statistically significantly lower risk of all-cause mortality $(95 \% \mathrm{Cl} 0.02-0.34)$ and arterial thromboembolic adverse events ( $95 \% \mathrm{Cl} 0.09-0.69)$ compared with those who were administered three injections.

The SAVE-AMD was a 1-year prospective, two center, randomized, double-masked, controlled, intervention trial, conducted in Switzerland, in which 24 patients with neovascular AMD were randomized to receive intravitreal injections of ranibizumab $(0.5 \mathrm{mg})$ or bevacizumab $(1.25 \mathrm{mg})$ and 26 patients with dry AMD were included in the control group [75]. The innovation in this study was the primary endpoint, to determine if the intraocular treatment with anti-VEGF could change systemic endothelial function. Three of the 50 patients, who were all treated with anti-VEGF, had systemic thromboembolic adverse events: one case of fatal stroke, one pulmonary embolism, and one suspected transient ischemic attack. These events led to interruption of the study, although the safety monitory board could neither prove nor exclude a causal relationship. The authors did not provide further information on which of the anti-VEGF treatments led to the three cases of thromboembolic adverse events.
Recent meta-analysis evaluated the relative risk of systemic adverse events between bevacizumab and ranibizumab. Mikacic and Bosnar have published a meta-analysis study on intravitreal bevacizumab and cardiovascular risk in wet-AMD patients. Again, in this study, no significant evidence emerged that bevacizumab may increase the cardiovascular risks of patients with AMD [76].

A Cochrane review led by Moja investigated six multi-center clinical trials and three smaller-scale studies involving 3665 patients. The combined risk ratio for one or more systemic adverse events was $1.08,95 \% \mathrm{Cl}(0.90,1.31)$. Similar to the researchers conducting previous meta-analyses, Moja et al. concluded that there was no strong evidence of a difference in risk but that the data available were not sufficient to rule out clinically important differential risks, particularly for specific adverse events [77].

\subsection{Aflibercept}

Aflibercept (also named VEGF Trap-eye) is the most recent anti-VEGF drug that has been approved by the FDA in November 2011. Two equivalent Phase III clinical trials of VEGF Trap-eye VIEW 1 and VIEW 2 (VEGF Trap-eye Investigation of Efficacy and Safety in Wet AMD) were successfully conducted to assess if VEGF Trap-eye was not inferior and clinically equivalent to ranibizumab that is considered the standard against which all other drugs should be compared $[78,79]$. A total of 2457 patients were enrolled and randomized in two arms of treatment: intravitreal $0.5 \mathrm{mg}$ or $2 \mathrm{mg}$ aflibercept monthly, $2 \mathrm{mg}$ aflibercept every 2 months after three initial monthly doses (every 8 weeks after monthly injection for the first year, followed by pro re nata regimen for the second year) or $0.5 \mathrm{mg}$ ranibizumab. At 1-year follow up, no statistically significant difference in thromboembolic adverse events was reported between aflibercept and ranibizumab treatments $(2.3 \%$ for aflibercept, and $1.5 \%$ for ranibizumab). At 96-week follow up (2 years), no difference was reported, with the incidence of adverse events at $3.2 \%$ and $3.6 \%$ for aflibercept and ranibizumab, respectively, and $0.8 \%$ nonfatal strokes in both treatment arms. Considering together, the incidence of arterial thromboembolic events across all arms was $2.4 \%$ to $3.8 \%$ [80]. Despite the higher binding affinity for ill VEGF-a, the greater in vitro potency of aflibercept and its greater systemic absorption and the potential risk of systemic events has not been verified in any way.

\subsection{Conbercept and abicipar pegol}

The effects of two new anti-VEGF drugs were investigated in two prospective studies: conbercept and abicipar Pegol. The efficacy and safety of conbercept were studied by the POENIX study [81], a multicenter, double-masked, sham-control, phase III study in which patients with CNV secondary to AMD received intravitreal injections of conbercept $(0.5 \mathrm{mg})$ or sham injections, with 2:1 ratio (8 patients vs. 43 patients). Among the 124 patients enrolled, 113 (91.1\%) completed the 12-month study. At the 12-month endpoint, in the 
conbercept group, there were 42 (51.9\%) ocular adverse events and 46 (56.8\%) non-ocular adverse events. In the sham group, there were 22 (52.4\%) ocular adverse events and 19 (45.2\%) non-ocular adverse events. In both groups, the most common ocular adverse event was hemorrhage at the injection site (22 patients, $27.2 \%$, in the conbercept group vs. 12 patients, $28.6 \%$, in the sham group). The incidence of others ocular adverse events were increased intraocular pressure $(6.2 \%$ vs. $11.9 \%)$, reduced VA ( $8.6 \%$ vs. $2.4 \%)$, and vitreous floaters (3.7\% vs. $2.4 \%$ ) in conbercept and sham groups, respectively.

In the conbercept group, there was also a single case of cataract, macular hemorrhage, macular edema, retina edema, retinal hemorrhage, and allergic conjunctivitis. In the sham group, there was 1 case of retinal detachment, none in the other group. In both, there were no detected cases of endophthalmitis or uveitis. The incidence of arterothrombolic events was $1.2 \%$ (1 case) of anterior myocardial infarction in the conbercept group and $2.4 \%$ (1 case) of mild cerebral infaaction in the sham group. No systemic allergic reaction was reported and no death occurred during the trial period, but further studies are needed to prove its safety and efficacy.

The abicipar pegol has been tested in phase 2 REACH stage III study. This study was a 20-week, multicenter, randomized, parallel group, double-masked comparison in which patients with neovascular AMD received a total of three injections of abicipar $1 \mathrm{mg}$ or $2 \mathrm{mg}$ or five injections of Ranibizumab $0.5 \mathrm{mg}$ [82]. A total of 64 patients were enrolled, randomized in 3:3:2 ratios (25 abicipar, $1 \mathrm{mg} ; 23$ abicipar, $2 \mathrm{mg} ; 16$ ranibizumab, $0.5 \mathrm{mg}$ ); during the 20-week of study, none of the patients experienced ATEs. No cases of endophthalmitis or serious adverse events were reported. Overall, adverse events were reported in 15 patients out of 25 in the abicipar $1 \mathrm{mg}$ arm; 10 patients out of 23 in the abicipar $2 \mathrm{mg}$ arm, and 9 patients out of 16 in the ranibizumab $0.5 \mathrm{mg}$ arm.

The most common ocular adverse events were vitreous floaters (experienced in five patients: three in the abicipar $1 \mathrm{mg}$ group; 1 in the abicipar $2 \mathrm{mg}$ group; 1 in the ranibizumab $0.5 \mathrm{mg}$ group), vitreous detachment (four patients; two in the abicipar $1 \mathrm{mg}$ group and two in the abicipar $2 \mathrm{mg}$ group), and retinal hemorrhage (five patients; three in the abicipar $1 \mathrm{mg}$ group and two in the ranibizumab $0.5 \mathrm{mg}$ group). Furthermore, two cases of macular scar were reported, both in the ranibizumab $0.5 \mathrm{mg}$ group. These, together with four of the five total cases of retinal hemorrhages, were unrelated with drug or injections. However, the other case of retinal hemorrhage, in the abicipar $1 \mathrm{mg}$ arm, was related to the injection. All the adverse events were resolved without sequelae. The incidence of adverse events was reported to be similar between abicipar (both $1 \mathrm{mg}$ and $2 \mathrm{mg}$ ) and Ranibizumab (0.5 mg).

Similar to conbercept, abicipar needs to be tested in future studies to evaluate its safety profile and efficacy.

\section{Discussion}

In a prospective cohort study comprising over 10,405 persons between the ages of 49 and 73 years, Wong concluded that middle-aged persons with signs of early-stage AMD have a higher risk for stroke, independent of traditional stroke risk factors. There were 508 cases of AMD in the cohort and over a 10-year period, 241 persons had an ATE.

After adjusting for age, sex, ethnicity, and site, the authors found that persons with early-stage AMD had a higher cumulative annual incidence of ATEs than those without the disease $(4.08 \%$ vs. $2.14 \%)$ [83]. For these reasons, the use of drugs potentially favoring ATEs in persons affected by wet AMD has caused concern.

From the biological point of view, it has been shown that all anti-VEGF drugs injected into the vitreous chamber pass into the systemic circulation and are able to inhibit serumcirculating VEGF. The close repetition (monthly) of the injections can accumulate some anti-VEGF compounds and in theory, can increase the risk of ATEs.

Data obtained from small groups of patients seem to elucidate that the anti-VEGF drugs that contain the Fc antibody region (bevacizumab and aflibercept) can remain in circulation for a long time in concentrations close to their respective IC 50 [10].

The FC fragment of the antibody interacts with the FC receptor of endothelial cells facilitating the translocation of the antibody through the retinal blood barrier into the systemic circulation [84].

Additionally, the anti-VEGF drugs containing an FC region demonstrate a longer systemic half-life because the FC-receptor interaction protects the molecule from systemic catabolism [85]. This plausibly increases the risk of ATEs particularly with the use of bevacizumab and aflibercept compared to when ranibizumab is used.

However, contradictory to the pharmacokinetic data, clinical data have never shown that ranibizumab is safer than bevacizumab or aflibercept.

A Cochrane review that specifically evaluated the systemic safety of bevacizumab versus ranibizumab found no clinically or statistically significant differences in death or ATEs incidence between the two drugs after 2 years of follow-up [85].

The analysis of 10 head-to-head trials of bevacizumab versus ranibizumab conducted by Solomon et al. in 2019 confirmed the little or no differences in outcomes between the two drugs, both in terms of efficacy and rate of the ATEs [47].

Thus, although it has been shown that the systemic use of parenteral anti-VEGF, at high doses, significantly increases the ATEs cases, at the intraocular doses, this has not been demonstrated by any clinical study so far concluded.

It has never been shown that the incidence of stroke in AMD patients has changed since the introduction of anti-VEGF drugs.

A population-based study by the Mayo Clinic in Rochester showed that intravitreal anti-VEGF therapy [Pegaptanib $\mathrm{n}=$ 10 pts, bevacizumab $n=377$ pts, ranibizumab $n=72$ pts, or Aflibercept $n=45 p t s]$, does not increase the risk of stroke, myocardial infarction, or death in patients with wet-AMD, This retrospective study included 504 patients who had received intravitreal injection for wet-AMD and compared them with three control groups, corresponding in age and sex, who had not received treatment: one compound from patients suffering from dry AMD $(n=504)$, one from wet 
AMD diagnosed in the period preceding the use of these drugs ( $n=473)$, and one without maculopathy ( $n=504)$. Five years later, the risk of stroke and myocardial infarction in the patients were found to be $7.2 \%$ and $6.1 \%$, respectively. These data were similar to those of the control groups. Therefore, this study indicates the cardiovascular safety of intravitreal administration of anti-VEGF in patients with $A M D$ and that cardiac events affecting these patients are not attributable to treatment but to their higher cardiovascular risk [86].

To date, clinical studies on the analysis of the safety of antiVEGF drugs in patients with AMD have not conclusively demonstrated an increased risk of stroke, major cardiovascular events, or mortality compared to controls, although the study entry bias and low number of events may have resulted in inaccuracy in the calculation of the probability.

It is noteworthy that, many of the RCTs addressing the thromboembolic role of anti-VEGF drugs, excluded patients with histories of stroke, myocardial infarction and other cardiovascular diseases, though in clinical settings many patients have a clinical history including such conditions. In these RCTs, the systemic thromboembolic risk could be underestimated and complicate the systemic safety evaluation of anti-VEGF therapy in AMD patients.

Indeed, the massive use of anti-VEGF at $10-15 \mathrm{mg} / \mathrm{kg}$ in the treatment of solid metastatic cancer exerts adverse thromboembolic effects in $4-12 \%$ of pts (4-12\%) [87]. This is, however, a rather low percentage considering the serious pathology affecting these patients. The massive lowering of circulating VEGF should theoretically cause thromboembolic effects in all patients since VEGF is the most important factor for the survival of systemic vessels; however, it is probably not the only such factor. There may be production of additional survival factors or other systems, alternative to VEGF, that guarantee the survival of the endothelium of the vessels in humans. In addition to VEGF, other factors such as epidermal growth factor, endostatins, and galectins, which are produced by platelets or the endothelium, can influence the pro-angiogenic response to an ischemic condition [88].

Pharmacokinetic data also show a reduction in circulating VEGF, which does not necessarily correspond to the local dose of VEGF necessary for survival of the vascular endothelium. The low doses of anti-VEGF injected intravitreally seems to be inadequate to interfere with systemic VEGF actions, for example, on cardiac endothelium or brain vessels in most patients [10]. Most patients with AMD may also have the capacity to activate these alternative endothelial repair pathways that guarantee a lower susceptibility to ATEs.

Patients with a history of recent myocardial infarction or stroke or those with diabetic macular edema may be more susceptible to the negative consequences of the systemic inhibition of VEGF; however, this information has not been adequately proven. The clinical problem is that it is not possible, at the moment, to determine the type of patient who would be more susceptible to these consequences.

\section{Conclusion}

Anti-VEGF drugs have revolutionized treatment of wet-AMD improving quality of life of patients. VEGF exerts systemically plenty of rules especially on cardiovascular system. Massive systemic inhibition of VEGF could lead to cardiovascular adverse events as demonstrated in many RCTs conducted on patients treated for solid tumors with these molecules. The rising question is if intravitreal administration of small doses of the same drugs in patients with wAMD could lead to same adverse events, in particular, thromboembolic ones. Local administration of anti-VEGF drugs reduces systemic distribution of these molecules but small concentrations could be found in peripheral blood and in some cases above the $I C_{50}$.

Trials conducted on this cue have shown a good risk profile and no significant association between IVT administration of anti-VEGF and CV diseases but, the number of cardiovascular adverse events impedes to declare its complete safety. Given the baseline risk of thromboembolic events in AMD population, other studies should be conducted involving many more patients than in preceding trials. RCTs conducted failed to demonstrate any significant difference in term of ATE between anti-VEGF molecules but no study address this cue specifically. It could be noticed that almost all trials excluded patients with cardiovascular disease stories. Further studies should be conducted including these patients could be useful to obtain more significant results.

\section{Expert opinion}

Head-to-head trials provided evidence of clinical equivalence between ranibizumab, bevacizumab, and aflibercept.

The companies that make bevacizumab had never shown any scientific interest in organizing large multicenter studies to verify the clinical efficacy and safety of this drug. For this reason, we have fewer data on the safety of bevacizumab despite being the most used drug because it is extremely cheaper.

Despite the evidence that bevacizumab is effective in macular degeneration and despite the lack of obvious safety differences in unbiased studies between bevacizumab and other anti-VEGF drugs, some short case series and limited laboratory studies have highlighted its possible systemic danger and discouraged its use for macular degeneration treatment.

These data showed that bevacizumab and aflibercept, that contain the Fc antibody region, remain in circulation for a long time in concentrations close to their respective IC 50 [10].

In theory this could increase the risk of ATEs. However, it does not happen in the reality of the clinic and no comparative study demonstrated that ranibizumab is safer than bevacizumab and aflibercept. The analysis of 10 head-to-head trials of bevacizumab versus ranibizumab conducted by Solomon et al. in 2019 confirmed the little or no differences in outcomes between the two drugs, both in terms of efficacy and rate of the ATEs [47].

The arteriothrombotic risk appears sufficiently low when compared with the natural incidence of arteriothrombotic 
events in this category of elderly patients and acceptable if balanced with the great advantage for vision.

In our opinion, the magnitude of this RR would not seem significant compared to the economic advantage offered by bevacizumab, especially in developing countries.

However, it is appropriate to have a prudent attitude in the approach to these therapies. In fact, the detection of rare adverse events has not been the focus of many studies, and the current data available are insufficient. Many of the RCTs addressing the thromboembolic role of anti-VEGF drugs, excluded patients with histories of stroke, myocardial infarction and other cardiovascular diseases, though in clinical settings many patients have a clinical history including such conditions. In these RCTs, the systemic thromboembolic risk could be underestimated and complicate the systemic safety evaluation of anti-VEGF therapy in AMD patients.

Thus for patients with a history of recent thromboembolic events or myocardial infarction, it could be safer to use antiVEGF drugs with short systemic half-life and low $\mathrm{IC}_{50}$, and those that do not accumulate in the serum-like ranibizumab or pegaptanib. In case of prolonged therapies, it may also be advisable to alternate drugs with longer plasma half-lives, which have the FC fragment, with drugs with short plasma half-lives, without FC fragment. Clinicians should also try to minimize the dose and frequency of injections without compromising functional results, for example, by adopting the treat and extend or pro-re-nata paradigms rather than adopting a rigid monthly protocol. Support in this direction could be given by the most recent anti-VEGF drugs Abicipar Pegol, which can be administered after the loading phase every 3 months.

An important area of research is also the analysis of the use of anti-VEGF agents in combination with other treatments such as verteporfirin photodynamic therapy, intravitreal steroids, and systemic or local anti-inflammatory drugs in order to reduce the rate of intravitreal injections.

Further studies are needed on the safety of therapy in patients with a known history of ATEs in the last 3 months. The clinician should be aware of the potential cardiovascular risk and decide case-by-case in agreement with the patient on the type and frequency of treatment to be performed.

\section{Funding}

This paper was not funded.

\section{Declaration of interest}

The author has no relevant affiliations or financial involvement with any organization or entity with a financial interest in or financial conflict with the subject matter or materials discussed in the manuscript. This includes employment, consultancies, honoraria, stock ownership or options, expert testimony, grants or patents received or pending, or royalties.

\section{Reviewer disclosures}

Peer reviewers on this manuscript have no relevant financial or other relationships to disclose.

\section{References}

Papers of special note have been highlighted as either of interest $(\cdot)$ or of considerable interest $(\cdot \bullet)$ to readers.

1. Ozaki E, Campbell M, Kiang AS, et al. Inflammation of age related macular degeneration. Adv Exp Med Bio. 2014;801:229-235.

2. Wong WL, Su X, Li X, et al. Global prevalence of age-related macular degeneration and disease burden projection for 2020 and 2040: a systematic review and meta-analysis. Lancet Glob Health. 2014;2(2):106-116.

3. Bakri SJ, Thorne JE, Ho AC, et al. Safety and efficacy of anti-vascular endothelial growth factor therapies for neovascular age-related macular degeneration: a report by the American academy of ophthalmology. Ophthalmology. 2019;126(1):55-63.

4. Zarbin MA. Anti-VEGF agents and the risk of arteriothrombotic events. Asia Pac J Ophthalmol (Phila). 2018;7(1):63-67.

5. Lee JH, Canny MD, De Erkenez A, et al. A therapeutic aptamer inhibits angiogenesis by specifically targeting the heparin binding domain of VEGF165. Proc Natl Acad Sci USA. 2005;102 (52):18902-18907.

6. Goldbaum M, Cunningham M. Aptamers and intramers: pegaptanib. In: Retin Pharmacother. 2010. p. 265-272.

7. Macugen - pfizer: one of the world's premier biopharmaceutical companies www.pfizer.com/products/rx_product_macugen.jsp;.

8. Rosenfeld PJ, Brown DM, Heirer JS, et al. MARINA study group. ranibizumab for neovascular age-related macular degeneration. N Engl J Med. 2006;355(14):1419-1431.

- Very important because discuss results of large prospective studies on efficacy and safety of the drugs examined.

9. Brown DM, Michels M, Kaiser PK, et al. ANCHOR study group. ranibizumab versus verteporfin for neovascular age-related macular degeneration. N Engl J Med. 2006; 5; 355(14):1432-1444.

- Very important because discuss results of large prospective studies on efficacy and safety of the drugs examined.

10. Avery LR, Castellarin AA, Steinle NC, et al. Systemic pharmacokinetics and pharmacodynamics of intravitreal aflibercept, bevacizumab, and ranibizumab. Retina. 2017;37(10):1847-1858.

11. Kazazi-Hyseni L, Beinjein JH, Schellens JHM. Bevacizumab. Oncologist. 2010;15(8):819-825.

12. Aisenbrey S, Ziemssen F, Volker M, et al. Intravitreal bevacizumab (Avastin) for occult choroidal neovascularisation in age-related macular degeneration. Graefe's Arch Clin Exp Ophthalmol. 2007;245:941-948.

13. Heiduschka P, Fietz $\mathrm{H}$, Hofmeister $\mathrm{S}$, et al. Penetration of bevacizumab through the retina after intravitreal injection in the monkey. Invest Ophthalmol Vis Sci. 2007;48:2814-2823.

14. Ziemssen F, Zhu Q, Peters S, et al. Intensified monitoring of circadian blood pressure and heart rate before and after intravitreous injection of bevacizumab: preliminary findings of a pilot study. Int Ophthalmol. 2009;29(4):213-224.

15. Ranibizumab intravitreal injection. Initial U.S. Approval: 2006. 2006;4-33. [cited 2019 July 8]. Available from: https://www.access data.fda.gov/drugsatfda_docs/label/2014/125156s105lbl.pdf

16. $\mathrm{Yu}$ L, Liang $\mathrm{XH}$, Ferrara N. Comparing protein VEGF inhibitors: in vitro biological studies. Biochem Biophys Res Commun. 2011;408:276-281.

17. Holash J, Davis S, Papadopoulos N, et al. VEGF-Trap: a VEGF blocker with potent antitumor effects. Proc Natl Acad Sci USA. 2002;99 (17):11393-11398.

18. Semeraro F, Morescalchi F, Duse S, et al. Aflibercept in wet AMD: specific role and optimal use. Drug Des Devel Ther. 2013;7:711-722.

- Important because offers a comprehensive overview on the efficacy and safety profile of Aflibercept.

19. Dixon JA, Oliver OJL, Mandava N. VEGF trap-eye for the treatment of age-related macular degeneration. Expert Opin Investig Drug. 2009;18(10):1573-1580.

20. Li X, Xu G, Wang $Y$, et al. Safety and efficacy of conbercept in neovascular age-related macular degeneration: results from a 12-month randomized phase 2 study: AURORA study. Ophthalmology. 2014;121(9):1740-1747. 
21. Li $H$, Lei $N$, Zhang $M$, et al. Pharmacokinetics of a long-lasting anti-VEGF fusion protein in rabbit. Exp Eye Res. 2012;97(1):154-159.

22. Zhang $M$, Yu D, Yang $C$, et al. The pharmacology study of a new recombinant human VEGF receptor-fc fusion protein on experimental choroidal neovascularization. Pharm Res. 2009;26 (1):204-210.

23. Vazquez-Lombardi R, Phan TG, Zimmermann C, et al. Challenges and opportunities for non-antibody scaffold drugs. Drug Discov Today. 2015;20:1271-1283.

24. Pluckthun A. Designed ankyrin repeat proteins (DARPins): binding proteins for research, diagnostics, and therapy. Annu Rev Pharmacol Toxicol. 2015;55:489-511.

25. Eggel A, Buschor P, Baumann MJ, et al. Inhibition of ongoing allergic reactions using a novel anti-IgEDARPin- Fc fusion protein. Allergy. 2011;66:961-968.

26. Souied EH, Devin F, Mauget-Fay"sse M, et al. Treatment of exudative age-related macular degeneration with a designed ankyrin repeat protein that binds vascular endothelial growth factor: a phase I/II study. Am J Ophthalmol. 2014;158:724-732.

27. Campochiaro PA, Channa R, Berger BB, et al. Treatment of diabetic macular edema with a designed ankyrin repeat protein that binds vascular endothelial growth factor: a phase I/II study. Am J Ophthalmol. 2013;155:697-704.

28. Papadopoulos N, Martin J, Ruan Q, et al. Binding and neutralization of vascular endothelial growth factor (VEGF) and related ligands by VEGF Trap, ranibizumab and bevacizumab. Angiogenesis. 2012;15:171-185.

29. Krohne TU, Liu Z, Holz FG, et al. Intraocular pharmacokinetics of ranibizumab following a single intravitreal injection in humans. Am J Ophthalmol. 2012;154:682-686.

30. Gragoudas ES, Adamis AP, Cunningham ET Jr, et al. VEGF inhibition study in ocular neovascularization clinical trial group. pegaptanib for neovascular age related macular degeneration. $\mathrm{N}$ Engl J Med. 2004;351:2805-2816.

31. VEGF Inhibition Study in Ocular Neovascularization (V.I.S.I.O.N.) Clinical Trial Group, Chakravarthy U, Adamis AP, Cunningham ET $\mathrm{Jr}$, et al. Year 2 efficacy results of 2 randomized controlled clinical trials of pegaptanib for neovascular age-related macular degeneration. Ophthalmology. 2006;113(9):1508.e1-25.

32. Singerman $L$, Masonson $H$, Patel $M$, et al. Pegaptanib sodium for neovascular age-related macular degeneration: third-year safety results of the VEGF Inhibition Study in ocular neovascularisation (VISION) trial. Br J Ophthalmol. 2008;92(12):1606-1611.

33. Rinaldi $M$, Chiosi F, Dell'Omo R, et al. Intravitreal pegaptanib sodium (Macugen) for treatment of myopic choroidal neovascularization: a morphologic and functional study. Retina. 2013;33(2):397-402.

34. Rinaldi M, Chiosi F, dell'Omo R, et al. Intravitreal pegaptanib sodium (Macugen ${ }^{\oplus}$ ) for treatment of diabetic macular oedema: a morphologic and functional study. Br J Clin Pharmacol. 2012;74 (6):940-946.

35. van Wijngaarden $\mathrm{P}$, Coster DJ, Williams KA. Inhibitors of ocular neovascularization: promises and potential problems. JAMA. 2005;293:1509-1513.

36. Ko J, Ross J, Awad H, et al. The effects of ZD6474, an inhibitor of VEGF signaling, on cutaneous wound healing in mice. J Surg Res. 2005;129:251-259.

37. Battaglia Parodi M, Di Bartolo E, Brue C, et al. Pegaptanib: choroidal neovascularization in patients with age-related macular degeneration and previous arterial thromboembolic events. Eur J Ophthalmol. 2018;28(1):58-62.

38. Semeraro F, Morescalchi F, Duse S, et al. Pharmacokinetic and pharmacodynamic properties of anti-vegf drugs after intravitreal injection. Curr Drug Metab. 2015;16(7):572-584.

39. Holz FG, Amoaku W, Donate J, et al. SUSTAIN study group. safety and efficacy of a flexible dosing regimen of ranibizumab in neovascular age-related macular degeneration: the SUSTAIN study. Ophthalmology. 2011;118(4):663-671.

- Important because examines the safety profile of anti-VEGF agents.
40. Antoszyk AN, Tuomi L, Chung CY, et al. FOCUS study group. ranobizumab combined with verteporfin photodynaimc therapy in age-realted neovascular generation (FOCUS): year 2 results. Am J Ophthalmol. 2008;145(5):862-874.

.- Very important because discuss results of large prospective studies on efficacy and safety of the drugs examined.

41. Abraham P, Yue H, Wilson L. Randomized, double-masked, sham-controlled trial of ranibizumab for neovascular age-related macular degeneration: PIER study year2. Am J Ophthalmol. 2010;150(3):315-324.

42. Schmidt-Erfurth U, Eldem B, Guymer R, et al. Efficacy and safety of monthly versus quarterly ranibizumab treatment in neovascular age-related macular degeneration: the EXCITE study. Ophthalmology. 2011;118(5):831-839.

- Important because discuss safety of Ranibizumab

43. Mitchell P, Korobelnik JF, Lanzetta P, et al. Ranibizumab (Lucentis) in neovascular age-related macular degeneration: evidence from clinical trials. Br J Ophthalmol. 2010;94(1):2-13.

44. Chong NV. Should avastin be used to treat age-related macular degeneration in the NHS? No Eye (Lond). 2009;23:1250-1253.

45. Schmidt-Erfurth U. Clinical safety of ranibizumab in age-related macular degeneration. Expert Opin Drug Saf. 2010;9:149-165.

46. Boyer DS, Heier JS, Brown DM, et al. A phase IIIb study to evaluate the safety of ranibizumab in subjects with neovascular age-related macular degeneration. Ophthalmology. 2009;116(9):1731-1739.

47. Ciulla TA, Rosenfeld PJ. Antivascular endothelial growth factor therapy for neovascular age-related macular degeneration. CurrOpinOphthalmol. 2009;20(3):158-165.

48. CHMP. Ranibizumab (lucentis). Annex I summary of product characteristics. [cited 2019 July 8]. Available from: https://www.ema. europa.eu/en/documents/product-information/lucentis-epar-pro duct-information_en.pdf

49. Ueta T, Yanagi $Y$, Tamaki $Y$, et al. Cerebrovascular accidents in ranibizumab. Ophthalmology. 2009;116:362.

- Important because examines cerebrovascular risks connected with the use of Ranibizumab.

50. Singer MA, Awh CC, Sadda S, et al. HORIZON: an open-label extension trial of ranibizumab for choroidal neovascularization secondary to age-related macular degeneration. Ophthalmology. 2012;119 (6):1175-1183.

51. Silva R, Axer-Siegel R, Eldem $B$, et al. SECURE study group. the SECURE study: long-termsafety of ranibizumab $0.5 \mathrm{mg}$ in neovascular age-related macular degeneration. Ophthalmology. 2013;120 (1):130-139.

- Important because discuss safety of Ranibizumab.

52. Rakic J-M, Leys A, Brié $H$, et al. Real-world variability in ranibizumab treatment and associated clinical, quality of life, and safety outcomes over 24 months in patients with neovascular age-related macular degeneration: the HELIOS study. Clin Ophthalmol. 2013;7:1849-1858.

53. Igaki T TNS opinion and social. Special Eurobarometer. 2018. [cited 2019 July 8]. Available from: https://data.europa.eu/euodp/de/ data/dataset/S790 72 3 EBS332

54. LUMINOUS: study to observe the effectiveness and safety of ranibizumab through individualized patient treatment and associated outcomes. [cited 2019 July 8]. Available from: http://clinicaltrials. gov/show/NCT01318941.

55. Finger RP, Wiedemann P, Blumhagen F, et al. Treatment patterns, visual acuity and quality-of-life outcomes of the WAVE study a non interventional study of ranibizumab treatment for neovascular age-related macular degeneration in Germany. Acta Ophthalmol. 2013;91(6):540-546.

56. Ueta $T$, Noda $Y$, Toyama $T$, et al. Systemic vascular safety of ranibizumab for age-related macular degeneration: systematic review and meta-analysis of randomized trials. Ophthalmology. 2014;121 (11):2193-203.1-7.

57. Steinbrook R. The price of sight-ranibizumab, bevacizumab, and the treatment of macular degeneration. N Engl J Med. 2006;355 (14):1409-1412. 
58. Semeraro F, Morescalchi F, Duse S, et al. Systemic thromboembolic adverse events in patients treated with intravitreal anti-VEGF drugs for neovascular age-related macular degeneration: an overview. Expert Opin Drug Saf. 2014;13(6):1-18.

59. Wu L, Martínez-Castellanos MA, Quiroz-Mercado $\mathrm{H}$, et al. for the Pan American Collaborative Retina Group (PACORES). twelve-month safety of intravitreal injections of bevacizumab (Avastin(R)): results of the Pan-American Collaborative Retina Study Group (PACORES). Graefes Arch Clin Exp Ophthalmol. 2008;246:81-87.

60. Hwang DJ, Kim YW, Woo SJ, et al. Comparison of systemic adverse events associated with intravitreal anti-VEGF injection: ranibizumab versus bevacizumab. J Korean Med Sci. 2012;27(12):1580-1585.

61. Biswas P, Sengupta S, Choudhary R, et al. Comparative role of intravitreal ranibizumab versus bevacizumab in choroidal neovascular membrane in age-related macular degeneration. Indian J Ophthalmol. 2011;59(3):191-196.

62. Brechner RJ, Rosenfeld PJ, Babish JD, et al. Pharmacotherapy for neovascular age-related macular degeneration: an analysis of the 100\% 2008 medicare fee-for-service part B claims file. Am J Ophthalmol. 2011;151(5):887-95e1.

63. Curtis LH, Hammill BG, Schulman KA, et al. Risks of mortality, myocardial infarction, bleeding, and stroke associated with therapies for age-related macular degeneration. Arch Ophthalmol. 2010;128(10):1273-1279.

64. Alexander SL, Linde-Zwirble WT, Werther W, et al. Annual rates of arterial thromboembolic events in medicare neovascular age-related macular degeneration patients. Ophthalmology. 2007;114(12):2174-2178.

65. Nguyen-Khoa BA, Goehring EL Jr, Werther W, et al. Hospitalized cardiovascular diseases in neovascular age-related macular degeneration. Arch Ophthalmol. 2008;126(9):1280-1286.

66. Campbell RJ, Gill SS, Bronskill SE, et al. Adverse events with intravitreal injection of vascular endothelial growth factor inhibitors: nested case-control study. Br Med J. 2012;345:e4203.

67. CATT Research Group, DF M, MG M, GS Y, et al. Ranibizumab and Bevacizumab for neovascular age-related macular degeneration. N Engl J Med. 2011;364:1897-1908.

.. Very important because discuss results of large prospective studies on efficacy and safety of drugs examined.

68. Chakravarthy U, Harding SP, Rogers CA, et al. IVAN Study Investigators. Alternative treatments to inhibit VEGF in age related choroidal neovascularisation: 2-year findings of the IVAN randomised controlled trial. Lancet. 2013;382:1258-1267.

-. Very important because discuss results of large prospective studies on efficacy and safety of the drugs examined.

69. Kodjikian L, Souied EH, Mimoun G, et al. GEFAL study group. ranibizumab versus bevacizumab for neovascular agerelated macular degeneration: results from the GEFAL noninferiority randomized trial. Ophthalmology. 2013;120:2300-2309.

70. Maguire MG, Martin DF, Ying GS, et al. Comparison of Age-related Macular Degeneration Treatments Trials (CATT) research group. five-year outcomes with anti-vascular endothelial growth factor treatment of neovascular age-related macular degeneration: the comparison of age-related macular degeneration treatments trials. Ophthalmology. 2016;123:1751-1761.

71. Ahfat FG, Zaidi FH. Bevacizumab vs ranibizumab-an appraisal of the evidence from CATT and IVAN. Eye (Lond). 2013 Mar;27(3):289-290.

72. Krebs I, Schmetterer L, Boltz A, et al. MANTA research group. a randomised double-masked trial comparing the visual outcome after treatment with ranibizumab or bevacizumab in patients with neovascular age-related macular degeneration. $\mathrm{Br} J$ Ophthalmol. 2013;97(3):266-271.
73. Maguire MG, Shaffer J, Ying G, et al. Serious adverse events with bevacizumab or ranibizumab for age-related macular degeneration: meta-analysis of individual patient data. Ophthalmol Retin. 2017;1(5):375-381.

74. Sangroongruangsri S, Chaikledkaew U, Kumluang S, et al. Realworld safety of intravotreal bevacizumab and ranibizumab treatments for retinal diseases in Thailand: a prospective obervational study. Clin Drug Investig. 2018;38:853-860.

75. Enseleit F, Michels S, Sudano I, et al. SAVE-AMD: safety of VEGF inhibitors in age-related macular degeneration. Ophthalmologica. 2017;238(4):205-216.

76. Mikačič I, Bosnar D. Intravitreal bevacizumab and cardiovascular risk in patients with age-related macular degeneration: systematic review and meta-analysis of randomized controlled trials and observational studies. Drug Saf. 2016;39:517-541.

77. Moja L, Lucenteforte $\mathrm{E}$, Kwag KH, et al. Systemic safety of bevacizumab versus ranibizumab for neovascular age-related macular degeneration. Cochrane Database Syst Rev. 2014;(9):CD011230.

78. Stewart MW, Rosenfeld PJ, Penha FM, et al. Pharmacokinetic rationale for dosing every 2 weeks versus 4 weeks with intravitreal ranibizumab, bevacizumab, and aflibercept (vascular endothelial growth factor trap-eye). Retina. 2012;32(3):343-457.

79. Heier JS, Brown DM, Chong V, et al. VIEW 1 and VIEW 2 Study Groups. Intravitreal aflibercept (VEGF trap-eye) in wet age-related macular degeneration. Ophthalmology. 2012;119(12):2537-2548. Erratum in: Ophthalmology. 2013;120(1):209-10.

-. Very important because discuss results of large prospective studies on efficacy and safety of the drugs examined.

80. Schmidt-Erfurth U, Kaiser PK, Korobelnik JF, et al. Intravitreal aflibercept injection for neovascular age-related macular geberation: ninty-six-week results of the VIEW studies. Ophthalmology. 2014;121:193-201.

.- Very important because discuss results of large prospective studies on efficacy and safety of the drugs examined.

81. Liu K, Song Y, Xu G, et al. Conbercept for treatment of neovascular age-related macular degeneration: results of the randomized phase 3 PHOENIX study. Am J Ophthalmol. 2019;197:156-167.

82. Callanan D, Kunimoto D, Maturi RK, et al. Double-masked, randomized, phase 2 evaluation of abicipar pegol (an Anti-VEGF DARPin Therapeutic) in neovascular age-related macular degeneration. J Ocul Pharmacol Ther. 2018;34(10):700-709.

83. Gentile R. More research is needed to support the association of AMD and stroke. SurvOphthalmol. 2007;52(5):551-553.

84. Zehetner C, Kirchmair R, Huber S, et al. Plasma levels of vascular endothelial growth factor before and after intravitreal injection of bevacizumab, ranibizumab and pegaptanib in patients with age-related macular degeneration, and in patients with diabetic macular oedema. Br J Ophthalmol. 2013;97(4):454-459.

85. Matsuyama K, Ogata N, Matsuoka M, et al. Plasma levels of vascular endothelial growth factor and pigment epithelium-derived factor before and after intravitreal injection of bevacizumab. $\mathrm{Br}$ J Ophthalmol. 2010;94(9):1215-1218.

86. Dalvin LA, Starr MR, AbouChehade JE, et al. Association of intravitreal anti-vascular endothelial growth factor therapy with risk of stroke, myocardial infarction, and death in patients with exudative age-related macular degeneration. JAMA Ophthalmol. 2019;137 (5):483.

87. Nalluri SR, Chu D, Keresztes $R$, et al. Risk of venous thromboembolism with the angiogenesis inhibitor bevacizumab in cancer patients: a meta-analysis. JAMA. 2008;300(19):2277-2285.

88. Etulan J, Negrotto S, Tribulatti MV, et al. Control of angiogenesis by galectins involves the release of platelet derived proangiogenic factors. PLoS One. 2014;9(4):e96402. 